Global Journal of Pure and Applied Mathematics.

ISSN 0973-1768 Volume 11, Number 4 (2015), pp. 2519-2535

(C) Research India Publications

https://dx.doi.org/10.37622/GJPAM/11.4.2015.2519-2535

\title{
Multivalued Intuitionistic Fuzzy Fractals
}

\author{
D. Easwaramoorthy ${ }^{1}$ \\ School of Advanced Sciences (Mathematics), \\ VIT University, Vellore - 632 014, Tamil Nadu, India. \\ R. Uthayakumar \\ Department of Mathematics, \\ The Gandhigram Rural Institute - Deemed University, \\ Gandhigram - 624 302, Dindigul, Tamil Nadu, India.
}

\begin{abstract}
In this paper, we initiate the notion of multivalued fractals in intuitionistic fuzzy metric spaces via iterated multifunction system, which consists of finite number of intuitionistic fuzzy B-contractions and intuitionistic fuzzy Edelstein contractions. Also we prove the existence and uniqueness of multivalued fractals in a complete intuitionistic fuzzy metric space and a compact intuitionistic fuzzy metric space. In addition to that, we explore the Collage Theorem to analyze the multivalued intuitionistic fuzzy fractals in the standard intuitionistic fuzzy metric spaces.
\end{abstract}

AMS subject classification: 26E50, 28A80, 47H10.

Keywords: Fractals, Intuitionistic Fuzzy B-Contraction, Intuitionistic Fuzzy Edelstein Contraction, Iterated Multifunction System, Intuitionistic Fuzzy Metric Space.

\section{Introduction}

Fuzzy set theory was introduced by Zadeh in 1965 [1]. The notion of intuitionistic fuzzy sets was defined by Atanassov in 1986 as a generalization of fuzzy sets [2]. Many authors have introduced and discussed several notions of fuzzy metric space in different ways $[3,4,5]$ and also proved fixed point theorems with interesting consequent results in the fuzzy metric spaces [6].

\footnotetext{
${ }^{1}$ Corresponding author.
} 
The concept of intuitionistic fuzzy metric space was given by Park [7] and the subsequent fixed point results in the intuitionistic fuzzy metric spaces are investigated by Alaca and et al. [8] and Mohamad [9]. Nowadays, the concepts of fuzzy metrics are explicitly used in the Image Processing as a main tool. The peer group concept in the impulsive noise detection in color images is redefined by means of a certain fuzzy metric. This concept is employed for the fast detection of noisy pixels by taking advantage of the fuzzy metric properties.

Fractal Analysis was introduced by Mandelbrot in 1975 [10] and popularized by various mathematicians $[11,12,13,14,15]$. Mandelbrot defined a Fractal mathematically as a set with Hausdorff dimension strictly exceeds its topological dimension [10]. Fractal sets are mathematical models of non-integer dimensional sets satisfying certain scaling properties. A mathematical fractal looks the same at all scales of magnification. This is an approximation to physical fractals which appears similar to the original object only for a certain range of scales. Such sets, when they have the additional property of being in some sense either strictly or statistically self-similar, have been used extensively by Mandelbrot and others to model various physical phenomena [10,11].

Fractals have broad applications in computer graphics, quantum physics, bio-medicine and other numerous areas of sciences. The Iterated Function System (IFS) was born as an application of the theory of discrete dynamical systems and is a useful tool to build fractal and other similar sets. Rodriguez-Lopez and Romaguera [16] provided a new contribution to the development of the theory of fuzzy metrics in a potentially interesting direction due to the undoubted importance of Hausdorff distance not only in general topology but also in other areas of Mathematics and Computer Science, such as fractals, image computing, etc.

Hutchinson [11] and Barnsley [12] initiated and developed the theory, called as Hutchinson-Barnsley theory (HB theory), in order to define and construct the fractal as a compact invariant subset of a complete metric space generated by the Iterated Function System (IFS) of contractions, by using the Banach fixed point theorem.

So, IFS have proven to be a very useful way of constructing self-similar or fractal objects on metric spaces. Several authors generalized and extended the HB theory by replacing the classical fixed point theorem by other metric and topological fixed point theorems in order to define metric fractals, topological fractals, Tarski's fractals, semifractals, multivalued fractals, etc. Especially in [17, 18, 19, 20, 21, 22]; multivalued fractals are developed in the metric and topological spaces. Also fuzzy fractal space and fuzzy fixed point theorems in fuzzy fractal spaces were constructed by Al-Saidi and et al $[23,24]$. Recently fractal concepts are introduced in fuzzy metric spaces as well as in intuitionistic fuzzy metric spaces and also proved the results for uniqueness and existence of Fractals with some motivating properties [25, 26, 27, 28, 29]. So far, there is no theory about multivalued fractals in intuitionistic fuzzy spaces. These directions have motivated us to construct the multivalued fractals in intuitionistic fuzzy metric spaces. So we intend to initiate the multivalued fractals in intuitionistic fuzzy spaces and to discuss some properties on it.

In this paper, we define the notion of multivalued fractals in intuitionistic fuzzy 
metric spaces via iterated multifunction system (IMS), which consists of finite number of intuitionistic fuzzy B-contractions and intuitionistic fuzzy Edelstein contractions. Also we prove some results to analyze the multivalued intuitionistic fuzzy fractals.

The organization of the paper is as follows: in Section 2, Preliminaries of Metric Fractals, Multivalued Metric Fractals, Intuitionistic Fuzzy Metric Spaces with required results are stated. In Section 3, Iterated Multifunction System and HB operator of Multivalued Intuitionistic Fuzzy B-Contractions and Multivalued Intuitionistic Fuzzy Edelstein Contractions are defined, and also existence and uniqueness theorem of Multivalued Intuitionistic Fuzzy Fractals on both complete and compact spaces are proved. The Collage theorem for Iterated Multifunction System of Multivalued Intuitionistic Fuzzy B-Contractions is discussed in Section 4. Finally, the concluding remarks are presented in Section 5.

\section{Preliminaries}

\subsection{Metric Fractals}

In this section, we recall the Hutchinson-Barnsley theory (HB theory) to define and construct the fractals in the complete metric space.

Definition 2.1. $[12,13]$ Let $(X, d)$ be a metric space and $\mathscr{K}_{o}(X)$ be the collection of all non-empty compact subsets of $X$.

Define, $d(x, B):=\inf _{y \in B} d(x, y)$ and $d(A, B):=\sup _{x \in A} d(x, B)$ for all $x \in X$ and $A, B \in \mathscr{K}_{o}(X)$. The Hausdorff metric or Hausdorff distance $\left(H_{d}\right)$ is a function $H_{d}$ : $\mathscr{K}_{o}(X) \times \mathscr{K}_{o}(X) \longrightarrow \mathbb{R}$ defined by

$$
H_{d}(A, B)=\max \{d(A, B), d(B, A)\} .
$$

Then $H_{d}$ is a metric on the hyperspace of compact sets $\mathscr{K}_{o}(X)$ and hence $\left(\mathscr{K}_{o}(X), H_{d}\right)$ is called a Hausdorff metric space.

Definition 2.2. $[11,12]$ Let $(X, d)$ be a metric space and $f_{n}: X \longrightarrow X, n=$ $1,2,3, \ldots, N_{o}\left(N_{o} \in \mathbb{N}\right)$ be $N_{o}$ - contraction mappings with the corresponding contractivity ratios $k_{n}, n=1,2,3, \ldots, N_{o}$. The system $\left\{X ; f_{n}, n=1,2,3, \ldots, N_{o}\right\}$ is called an Iterated Function System (IFS) or Hyperbolic Iterated Function System with the ratio $k=\max _{n=1}^{N_{o}} k_{n}$.

Then the Hutchinson-Barnsley operator (HB operator) of the IFS is a function $F$ : $\mathscr{K}_{o}(X) \longrightarrow \mathscr{K}_{o}(X)$ defined by

$$
F(B)=\bigcup_{n=1}^{N_{o}} f_{n}(B), \text { for all } B \in \mathscr{K}_{o}(X) .
$$

Further, the $H B$ operator $(F)$ is a contraction mapping on $\left(\mathscr{K}_{o}(X), H_{d}\right)$. 
Theorem 2.3. [HB Theorem for IFS $[11,12]]$ Let $(X, d)$ be a complete metric space and $\left\{X ; f_{n}, n=1,2,3, \ldots, N_{o} ; N_{o} \in \mathbb{N}\right\}$ be an IFS on $X$. Then, there exists only one compact invariant set $A^{*} \in \mathscr{K}_{o}(X)$ of the $H B$ operator $(F)$ or, equivalently, $F$ has a unique fixed point namely $A^{*} \in \mathscr{K}_{o}(X)$.

Definition 2.4. [12] The fixed point $A^{*} \in \mathscr{K}_{0}(X)$ of the $H B$ operator $F$ described in the Theorem 2.3 is called the Attractor (Fractal) of the IFS. Sometimes $A^{*} \in \mathscr{K}_{o}(X)$ is called as Fractal generated by the IFS and so called Metric Fractal.

\subsection{Multivalued Metric Fractals}

In this section, we recall the iterated multifunction system to define and construct the multivalued fractals in the complete metric space.

Definition 2.5. $[17,20,22]$ Let $(X, d)$ be a metric space and $f_{n}: X \longrightarrow \mathscr{K}_{0}(X), n=$ $1,2,3, \ldots, N_{o}\left(N_{o} \in \mathbb{N}\right)$ be $N_{o}$ - multivalued contractions (with non-empty compact values) with the corresponding contractivity ratios $k_{n}, n=1,2,3, \ldots, N_{o}$. i.e.,

$$
H_{d}\left(f_{n}(x), f_{n}(y)\right) \leq k_{n} d(x, y)
$$

for all $x, y \in X$ and $k_{n} \in[0,1) ; n=1,2,3, \ldots, N_{o}$.

The system $\left\{X ; f_{n}, n=1,2,3, \ldots, N_{o}\right\}$ is called an Iterated Multifunction System (IMS) with the ratio $k=\max _{n=1}^{N_{o}} k_{n}$.

The associated Hutchinson-Barnsley map (HB map) of the IMS is a function $F$ : $X \longrightarrow \mathscr{K}_{o}(X)$ defined by

$$
F(x)=\bigcup_{n=1}^{N_{o}} f_{n}(x), \text { for all } x \in X
$$

Then the induced Hutchinson-Barnsley operator (HB operator) of the IMS is a function $F^{*}: \mathscr{K}_{o}(X) \longrightarrow \mathscr{K}_{o}(X)$ defined by

$$
F^{*}(B)=\overline{\bigcup_{x \in B} F(x)}=\bigcup_{x \in B} F(x), \text { for all } B \in \mathscr{K}_{o}(X) .
$$

Theorem 2.6. [HB Theorem for Metric IMS $[17,20,22]]$ Let $(X, d)$ be a complete metric space and $\left\{X ; f_{n}, n=1,2,3, \ldots, N_{o} ; N_{o} \in \mathbb{N}\right\}$ be an IMS on $X$. Then, there exists exactly one compact invariant set $A^{*} \in \mathscr{K}_{o}(X)$ of the $H B$ operator $(F)$ or, equivalently, $F^{*}$ has a unique fixed point namely $A^{*} \in \mathscr{K}_{o}(X)$.

Definition 2.7. $[17,20,22]$ The fixed point $A^{*} \in \mathscr{K}_{o}(X)$ of the $H B$ operator $F^{*}$ described in the Theorem 2.6 is called the Multivalued Attractor (Multivalued Fractal) of the IMS. Sometimes $A^{*} \in \mathscr{K}_{o}(X)$ is called as Multivalued Fractal generated by the IMS and so called Multivalued Metric Fractal. 
Theorem 2.8. [Collage Theorem for Metric IMS [20]] Let $(X, d)$ be a complete metric space. Let $\left(\mathscr{K}_{o}(X), H_{d}\right)$ be the corresponding Hausdorff metric space and $\left\{X ; f_{n}, n=1,2,3, \ldots, N_{o} ; N_{o} \in \mathbb{N}\right\}$ be an IMS on $X$ with the contractivity ratio $k$. If $B \in \mathscr{K}_{o}(X)$, then

$$
H_{d}\left(A^{*}, B\right) \leq \frac{H_{d}\left(B, F^{*}(B)\right)}{1-k},
$$

where $F^{*}$ is the $H B$ operator and $A^{*}$ is the attractor of the IMS.

\subsection{Intuitionistic Fuzzy Metric Space}

In [1], Zadeh defined a fuzzy set on $X$ as a function $f: X \longrightarrow[0,1]$. In order to define and construct the intuitionistic fuzzy IFS fractals with respect to Hausdorff intuitionistic fuzzy metric by developing the HB theory, we state the required concepts of intuitionistic fuzzy metric space based on fuzzy sets, continuous t-norm and continuous t-conorm as follows:

Definition 2.9. $[7,8,30]$ A 5-tuple $(X, M, N, *, \diamond)$ is said to be an intuitionistic fuzzy metric space if $X$ is an arbitrary (non-empty) set, $*$ is a continuous t-norm [32], $\diamond$ is a continuous t-conorm [32] and $M, N$ are fuzzy sets on $X^{2} \times[0, \infty)$ satisfying the following conditions:

(a) $M(x, y, t)+N(x, y, t) \leq 1$;

(b) $M(x, y, 0)=0$;

(c) $M(x, y, t)=1$ if and only if $x=y$;

(d) $M(x, y, t)=M(y, x, t)$;

(e) $M(x, y, t) * M(y, z, s) \leq M(x, z, t+s)$;

(f) $M(x, y, \cdot):[0, \infty) \longrightarrow[0,1]$ is left continuous;

(g) $\lim _{t \rightarrow \infty} M(x, y, t)=1$;

(h) $N(x, y, 0)=1$;

(i) $N(x, y, t)=0$ if and only if $x=y$;

(j) $N(x, y, t)=N(y, x, t)$;

(k) $N(x, y, t) \diamond N(y, z, s) \geq N(x, z, t+s)$;

(l) $N(x, y, \cdot):[0, \infty) \longrightarrow[0,1]$ is right continuous;

(m) $\lim _{t \rightarrow \infty} N(x, y, t)=0$ 
for all $x, y, z \in X$ and $t, s>0$.

Then $(M, N, *, \diamond)$ or simply $(M, N)$ is called an intuitionistic fuzzy metric on $X$. The functions $M(x, y, t)$ and $N(x, y, t)$ represents the degree of nearness and the degree of non-nearness between $x$ and $y$ in $X$ with respect to $t$, respectively.

Definition 2.10. [7] Let $(X, d)$ be a metric space. Let $M_{d}$ and $N_{d}$ be the functions defined on $X^{2} \times[0, \infty)$ by

$$
M_{d}(x, y, t)=\frac{t}{t+d(x, y)} \text { and } N_{d}(x, y, t)=\frac{d(x, y)}{t+d(x, y)},
$$

for all $x, y \in X$ and $t>0$. Then $\left(X, M_{d}, N_{d}, *, \diamond\right)$ is an intuitionistic fuzzy metric space, which is called standard intuitionistic fuzzy metric space, and $\left(M_{d}, N_{d}\right)$ is called as the standard intuitionistic fuzzy metric induced by the metric $d$.

Definition 2.11. [7] Let $(X, M, N, *, \diamond)$ be an intuitionistic fuzzy metric space. The open ball $B(x, r, t)$ with center $x \in X$ and radius $r, 0<r<1$, with respect to $t>0$, is defined as

$$
B(x, r, t)=\{y \in X: M(x, y, t)>1-r, N(x, y, t)<r\} .
$$

Define

$$
\begin{aligned}
\tau_{(M, N)}= & \{A \subset X: \text { for each } x \in A, \text { there exists } t>0 \text { and } \\
& r \in(0,1) \text { such that } B(x, r, t) \subset A\} .
\end{aligned}
$$

Then $\tau_{(M, N)}$ is a topology on $X$ induced by an intuitionistic fuzzy metric $(M, N)$.

The topologies induced by the metric and the corresponding standard intuitionistic fuzzy metric are the same.

Definition 2.12. [7, 8] Let $(X, M, N, *, \diamond)$ be an intuitionistic fuzzy metric space. We say that $X$ is complete if every Cauchy sequence is convergent. It is called compact if every sequence contains a convergent subsequence.

\subsubsection{Hausdorff Intuitionistic Fuzzy Metric Space}

In [30], Gregori et al. defined the Hausdorff intuitionistic fuzzy metric on intuitionistic fuzzy hyperspace $\mathscr{K}_{o}(X)$ and constructed the Hausdorff intuitionistic fuzzy metric space as follows.

Definition 2.13. [30] Let $(X, M, N, *, \diamond)$ be an intuitionistic fuzzy metric space. We shall denote by $\mathscr{K}_{o}(X)$, the set of all non-empty compact subsets of $X$.

Define,

$$
M(x, B, t):=\sup _{y \in B} M(x, y, t), \quad M(A, B, t):=\inf _{x \in A} M(x, B, t)
$$




$$
\begin{gathered}
\text { and } \\
N(x, B, t):=\inf _{y \in B} N(x, y, t), N(A, B, t):=\sup _{x \in A} N(x, B, t)
\end{gathered}
$$

for all $x \in X$ and $A, B \in \mathscr{K}_{o}(X)$.

Then we define the Hausdorff intuitionistic fuzzy metric $\left(H_{M}, H_{N}, *, \diamond\right)$ as follows:

$$
\begin{gathered}
H_{M}(A, B, t)=\min \{M(A, B, t), M(B, A, t)\} \\
\text { and } \\
H_{N}(A, B, t)=\max \{N(A, B, t), N(B, A, t)\} .
\end{gathered}
$$

Here $\left(H_{M}, H_{N}\right)$ is an intuitionistic fuzzy metric on the hyperspace of compact sets, $\mathscr{K}_{o}(X)$, and hence $\left(\mathscr{K}_{o}(X), H_{M}, H_{N}, *, \diamond\right)$ is called a Hausdorff intuitionistic fuzzy metric space.

Theorem 2.14. [27] Let $(X, M, N, *, \diamond)$ be an intuitionistic fuzzy metric space. Then $\left(\mathscr{K}_{o}(X), H_{M}, H_{N}, *, \diamond\right)$ is complete if and only if $(X, M, N, *, \diamond)$ is complete.

Theorem 2.15. [27] Let $(X, M, N, *, \diamond)$ be an intuitionistic fuzzy metric space. Then $\left(\mathscr{K}_{o}(X), H_{M}, H_{N}, *, \diamond\right)$ is compact if and only if $(X, M, N, *, \diamond)$ is compact.

Proposition 2.16. [29] Let $(X, d)$ be a metric space. Then, the Hausdorff intuitionistic fuzzy metric $\left(H_{M_{d}}, H_{N_{d}}\right)$ of the standard intuitionistic fuzzy metric $\left(M_{d}, N_{d}\right)$ coincides with the standard intuitionistic fuzzy metric $\left(M_{H_{d}}, N_{H_{d}}\right)$ of the Hausdorff metric $\left(H_{d}\right)$ on $\mathscr{K}_{o}(X)$, i.e., $H_{M_{d}}(A, B, t)=M_{H_{d}}(A, B, t)$ and $H_{N_{d}}(A, B, t)=N_{H_{d}}(A, B, t)$ for all $A, B \in \mathscr{K}_{o}(X)$ and $t>0$.

Theorem 2.17. [29] Let $(X, M, N, *, \diamond)$ be an intuitionistic fuzzy metric space. Let $\left(\mathscr{K}_{o}(X), H_{M}, H_{N}, *, \diamond\right)$ be the corresponding Hausdorff intuitionistic fuzzy metric space. If $A, B, C, D \in \mathscr{K}_{o}(X)$, then

$$
\begin{aligned}
& H_{M}(A \cup B, C \cup D, t) \geq \min \left\{H_{M}(A, C, t), H_{M}(B, D, t)\right\} \\
& \quad \text { and } \\
& H_{N}(A \cup B, C \cup D, t) \leq \max \left\{H_{N}(A, C, t), H_{N}(B, D, t)\right\}
\end{aligned}
$$

for all $t>0$.

\section{Multivalued Intuitionistic Fuzzy Fractals}

In this section, we define the Intuitionistic Fuzzy IMS and Multivalued Intuitionistic Fuzzy Fractals in the intuitionistic fuzzy metric space. 
Definition 3.1. Let $(X, M, N, *, \diamond)$ be an intuitionistic fuzzy metric space and $\left(\mathscr{K}_{o}(X)\right.$, $\left.H_{M}, H_{N}, *, \diamond\right)$ is the corresponding Hausdorff intuitionistic fuzzy metric space. We say that the mapping $f: X \longrightarrow \mathscr{K}_{o}(X)$ is multivalued intuitionistic fuzzy B-contraction (multivalued intuitionistic fuzzy Sehgal contraction) if there exists $k \in(0,1)$ such that

$$
\begin{gathered}
H_{M}(f(x), f(y), k t) \geq M(x, y, t) \\
\text { and } \\
H_{N}(f(x), f(y), k t) \leq N(x, y, t)
\end{gathered}
$$

for all $x, y \in X$ and $t>0$.

Definition 3.2. Let $(X, M, N, *, \diamond)$ be an intuitionistic fuzzy metric space and $\left(\mathscr{K}_{o}(X)\right.$, $\left.H_{M}, H_{N}, *, \diamond\right)$ is the corresponding Hausdorff intuitionistic fuzzy metric space. We say that the mapping $f: X \longrightarrow \mathscr{K}_{o}(X)$ is multivalued intuitionistic fuzzy Edelstein contraction if

$$
\begin{aligned}
& H_{M}(f(x), f(y), \cdot)>M(x, y, \cdot) \\
& \text { and } \\
& H_{N}(f(x), f(y), \cdot)<N(x, y, \cdot)
\end{aligned}
$$

for all $x, y \in X$ such that $x \neq y$.

Definition 3.3. Let $(X, M, N, *, \diamond)$ be an intuitionistic fuzzy metric space and $\left(\mathscr{K}_{o}(X)\right.$, $\left.H_{M}, H_{N}, *, \diamond\right)$ be the corresponding Hausdorff intuitionistic fuzzy metric space. Let $f_{n}$ : $X \longrightarrow \mathscr{K}_{o}(X), n=1,2,3, \ldots, N_{o}\left(N_{o} \in \mathbb{N}\right)$ be $N_{o}$ - multivalued intuitionistic fuzzy B-contractions (respectively multivalued intuitionistic fuzzy Edelstein contractions) with the corresponding contractivity ratios $k_{n}, n=1,2,3, \ldots, N_{o}$.

Then the system $\left\{X ; f_{n}, n=1,2,3, \ldots, N_{o}\right\}$ is called a Intuitionistic Fuzzy Iterated Multifunction System (IF-IMS) of multivalued intuitionistic fuzzy B-contractions (respectively multivalued intuitionistic fuzzy Edelstein contractions) on intuitionistic fuzzy metric space $(X, M, N, *, \diamond)$.

The associated Intuitionistic Fuzzy Hutchinson-Barnsley map (IF-HB map) of the IF-IMS is a function $F: X \longrightarrow \mathscr{K}_{o}(X)$ defined by

$$
F(x)=\bigcup_{n=1}^{N_{o}} f_{n}(x), \text { for all } x \in X
$$

Then the induced Intuitionistic Fuzzy Hutchinson-Barnsley operator (IF-HB operator) of the IF-IMS is a function $F^{*}: \mathscr{K}_{o}(X) \longrightarrow \mathscr{K}_{o}(X)$ defined by

$$
F^{*}(B)=\overline{\bigcup_{x \in B} F(x)}=\bigcup_{x \in B} F(x), \text { for all } B \in \mathscr{K}_{o}(X) .
$$


Theorem 3.4. Let $(X, M, N, *, \diamond)$ be an intuitionistic fuzzy metric space. Let $\left(\mathscr{K}_{o}(X)\right.$, $\left.H_{M}, H_{N}, *, \diamond\right)$ be the corresponding Hausdorff intuitionistic fuzzy metric space. Suppose $\left\{X ; f_{n}, n=1,2,3, \ldots, N_{o} ; N_{o} \in \mathbb{N}\right\}$ is an IF-IMS of multivalued intuitionistic fuzzy B-contractions with the same contractivity ratio $k \in(0,1)$ for each $n \in\left\{1,2, \ldots, N_{o}\right\}$. Then the IF-HB operator $F^{*}$ of IF-IMS is an intuitionistic fuzzy B-contraction on $\left(\mathscr{K}_{o}(X), H_{M}, H_{N}, *, \diamond\right)$ with the ratio $k$, i.e., for all $A, B \in \mathscr{K}_{o}(X)$ and $k \in(0,1)$, the IF-HB operator $F^{*}$ satisfies

$$
H_{M}\left(F^{*}(A), F^{*}(B), k t\right) \geq H_{M}(A, B, t)
$$

and

$$
H_{N}\left(F^{*}(A), F^{*}(B), k t\right) \leq H_{N}(A, B, t) .
$$

Proof. Fix $t>0, k \in(0,1)$ and let $A, B \in \mathscr{K}_{o}(X)$. For each $n \in\left\{1,2, \ldots, N_{o}\right\}$,

$$
\begin{aligned}
& H_{M}\left(f_{n}(A), f_{n}(B), k t\right) \\
& \quad=\min \left\{M\left(f_{n}(A), f_{n}(B), k t\right), M\left(f_{n}(B), f_{n}(A), k t\right)\right\} .
\end{aligned}
$$

Now,

$$
\begin{aligned}
M\left(f_{n}(A), f_{n}(B), k t\right) & =\inf _{x \in A}\left\{\sup _{y \in B}\left\{M\left(f_{n}(x), f_{n}(y), k t\right)\right\}\right\} \\
& \geq \inf _{x \in A}\left\{\sup _{y \in B}\left\{H_{M}\left(f_{n}(x), f_{n}(y), k t\right)\right\}\right\} .
\end{aligned}
$$

Since $f_{n}$ is a multivalued intuitionistic fuzzy B-contraction,

$$
\begin{aligned}
M\left(f_{n}(A), f_{n}(B), k t\right) & \geq \inf _{x \in A}\left\{\sup _{y \in B}\{M(x, y, t)\}\right\} \\
& =M(A, B, t) .
\end{aligned}
$$

Similarly, $M\left(f_{n}(B), f_{n}(A), k t\right) \geq M(A, B, t)$. Hence, $H_{M}\left(f_{n}(A), f_{n}(B), k t\right) \geq M(A, B, t)$ for all $n \in\left\{1,2, \ldots, N_{o}\right\}$. Then by Theorem 2.17 and using the above inequality, we 
have

$$
\begin{aligned}
H_{M}\left(F^{*}(A), F^{*}(B), k t\right) & =H_{M}\left(\bigcup_{x \in A} F(x), \bigcup_{x \in B} F(y), k t\right) \\
& =H_{M}\left(\bigcup_{x \in A}^{N_{0}} f_{n}(x), \bigcup_{x \in B} \bigcup_{n=1}^{N_{0}} f_{n}(y), k t\right) \\
& =H_{M}\left(\bigcup_{n=1}^{N_{0}} f_{n \in A}(x), \bigcup_{n=1}^{N_{0}} \bigcup_{x \in B} f_{n}(y), k t\right) \\
& \geq \min _{n=1}^{N_{o}} H_{M}\left(\bigcup_{x \in A} f_{n}(x), \bigcup_{x \in B} f_{n}(y), k t\right) \\
& =\min _{n=1}^{N_{o}} H_{M}\left(f_{n}(A), f_{n}(B), k t\right) \\
\geq \min _{n=1} M(A, B, t) & \\
& =M(A, B, t) .
\end{aligned}
$$

Similarly, $H_{M}\left(F^{*}(A), F^{*}(B), k t\right)=H_{M}\left(F^{*}(B), F^{*}(A), k t\right) \geq M(B, A, t)$. Thus, $H_{M}\left(F^{*}(A), F^{*}(B), k t\right) \geq H_{M}(A, B, t)$. Likewise, we can prove that

$$
H_{N}\left(F^{*}(A), F^{*}(B), k t\right) \leq H_{N}(A, B, t) .
$$

This completes the proof.

Theorem 3.5. Let $(X, M, N, *, \diamond)$ be an intuitionistic fuzzy metric space. Let $\left(\mathscr{K}_{o}(X)\right.$, $\left.H_{M}, H_{N}, *, \diamond\right)$ be the corresponding Hausdorff intuitionistic fuzzy metric space. Suppose $\left\{X ; f_{n}, n=1,2,3, \ldots, N_{o} ; N_{o} \in \mathbb{N}\right\}$ is an IF-IMS of multivalued intuitionistic fuzzy Edelstein contractions. Then the IF-HB operator $F^{*}$ is an intuitionistic fuzzy Edelstein contraction on $\left(\mathscr{K}_{o}(X), H_{M}, H_{N}, *, \diamond\right)$, i.e., for all $A, B \in \mathscr{K}_{o}(X)$ such that $A \cap B=\emptyset$, the IF-HB operator $F^{*}$ satisfies,

$$
H_{M}\left(F^{*}(A), F^{*}(B), \cdot\right)>H_{M}(A, B, \cdot)
$$

and

$$
H_{N}\left(F^{*}(A), F^{*}(B), \cdot\right)<H_{N}(A, B, \cdot) .
$$

Proof. Let $A, B \in \mathscr{K}_{o}(X)$ such that $A \cap B=\emptyset$. For each $n \in\left\{1,2, \ldots, N_{o}\right\}$,

$$
\begin{aligned}
& H_{M}\left(f_{n}(A), f_{n}(B), \cdot\right) \\
& \quad=\min \left\{M\left(f_{n}(A), f_{n}(B), k t\right), M\left(f_{n}(B), f_{n}(A), \cdot\right)\right\} .
\end{aligned}
$$


Now,

$$
\begin{aligned}
M\left(f_{n}(A), f_{n}(B), \cdot\right) & =\inf _{x \in A}\left\{\sup _{y \in B}\left\{M\left(f_{n}(x), f_{n}(y), \cdot\right)\right\}\right\} \\
& >\inf _{x \in A}\left\{\sup _{y \in B}\left\{H_{M}\left(f_{n}(x), f_{n}(y), \cdot\right)\right\}\right\}
\end{aligned}
$$

Since $f_{n}$ is a multivalued intuitionistic fuzzy Edelstein contraction and $A \cap B=\emptyset$,

$$
\begin{aligned}
M\left(f_{n}(A), f_{n}(B), \cdot\right) & >\inf _{x \in A}\left\{\sup _{y \in B}\{M(x, y, \cdot)\}\right\} \\
& =M(A, B, \cdot) .
\end{aligned}
$$

Similarly, $M\left(f_{n}(B), f_{n}(A), \cdot\right)>M(A, B, \cdot)$. Hence, $H_{M}\left(f_{n}(A), f_{n}(B), \cdot\right)>M(A, B, \cdot)$ for all $n \in\left\{1,2, \ldots, N_{o}\right\}$. Then by Theorem 2.17 and using the above inequality, we have

$$
\begin{aligned}
H_{M}\left(F^{*}(A), F^{*}(B), \cdot\right) & =H_{M}\left(\bigcup_{x \in A} F(x), \bigcup_{x \in B} F(y), \cdot\right) \\
& =H_{M}\left(\bigcup_{x \in A} \bigcup_{n=1}^{N_{0}} f_{n}(x), \bigcup_{x \in B} \bigcup_{n=1}^{N_{0}} f_{n}(y), \cdot\right) \\
& =H_{M}\left(\bigcup_{n=1}^{N_{0}} \bigcup_{x \in A} f_{n}(x), \bigcup_{n=1}^{N_{0}} \bigcup_{x \in B} f_{n}(y), \cdot\right) \\
& >\min _{n=1}^{N_{o}} H_{M}\left(\bigcup_{x \in A} f_{n}(x), \bigcup_{x \in B} f_{n}(y), \cdot\right) \\
& =\min _{n=1}^{N_{o}} H_{M}\left(f_{n}(A), f_{n}(B), \cdot\right) \\
& >\min _{n=1}^{N_{o}} M(A, B, \cdot) \\
& =M(A, B, \cdot) .
\end{aligned}
$$

Similarly, $H_{M}\left(F^{*}(A), F^{*}(B), \cdot\right)=H_{M}\left(F^{*}(B), F^{*}(A), \cdot\right)>M(B, A, \cdot)$. Thus, $H_{M}\left(F^{*}(A), F^{*}(B), \cdot\right)>H_{M}(A, B, \cdot)$. Likewise, we can prove that

$$
H_{N}\left(F^{*}(A), F^{*}(B), \cdot\right)<H_{N}(A, B, \cdot) .
$$

This completes the proof.

Theorem 3.6. [HB Theorem for IF-IMS in the Complete Intuitionistic Fuzzy Metric Space] Let $(X, M, N, *, \diamond)$ be a complete intuitionistic fuzzy metric space. Let 
$\left\{X ; f_{n}, n=1,2,3, \ldots, N_{o} ; N_{o} \in \mathbb{N}\right\}$ be an IF-IMS of multivalued intuitionistic fuzzy B-contractions and $F^{*}$ be the IF-HB operator of the IF-IMS. Then, there exists only one compact invariant set $A^{*} \in \mathscr{K}_{o}(X)$ of the IF-HB operator $\left(F^{*}\right)$ or, equivalently, $F^{*}$ has a unique fixed point namely $A^{*} \in \mathscr{K}_{o}(X)$.

Proof. Since $(X, M, N, *, \diamond)$ is a complete intuitionistic fuzzy metric space and by Theorem 2.14, we have $\left(\mathscr{K}_{o}(X), H_{M}, H_{N}, *, \diamond\right)$ is also complete Hausdorff intuitionistic fuzzy metric space. Also Theorem 3.4 shows that the IF-HB operator $F^{*}$ is an intuitionistic fuzzy B-contraction. Then by the Intuitionistic Fuzzy Banach Contraction Theorem (Theorem 7 in [8]), we conclude that $F^{*}$ has a unique fixed point, namely $A^{*} \in \mathscr{K}_{o}(X)$.

Theorem 3.7. [HB Theorem for IF-IMS in the Compact Intuitionistic Fuzzy Metric Space] Let $(X, M, N, *, \diamond)$ be a compact intuitionistic fuzzy metric space. Let $\left\{X ; f_{n}, n=1,2,3, \ldots, N_{o} ; N_{o} \in \mathbb{N}\right\}$ be an IF-IMS of multivalued intuitionistic fuzzy Edelstein contractions and $F^{*}$ be the IF-HB operator of the IF-IMS. Then, there exists only one compact invariant set $A^{*} \in \mathscr{K}_{o}(X)$ of the IF-HB operator $\left(F^{*}\right)$ or, equivalently, $F^{*}$ has a unique fixed point namely $A^{*} \in \mathscr{K}_{o}(X)$.

Proof. Since $(X, M, N, *, \diamond)$ is a compact intuitionistic fuzzy metric space and by Theorem 2.15 , we have $\left(\mathscr{K}_{o}(X), H_{M}, H_{N}, *, \diamond\right)$ is also compact Hausdorff intuitionistic fuzzy metric space. Also Theorem 3.5 shows that the IF-HB operator $F^{*}$ is an intuitionistic fuzzy Edelstein contraction. Then by the Intuitionistic Fuzzy Edelstein Contraction Theorem (Theorem 10 in [8]), we conclude that $F^{*}$ has a unique fixed point, namely $A^{*} \in \mathscr{K}_{o}(X)$.

Definition 3.8. We define the Multivalued Intuitionistic Fuzzy Attractor or Fractal on complete space (respectively compact space) as the set $A^{*} \in \mathscr{K}_{o}(X)$, which is described in the HB Theorem 3.6 for complete space (respectively HB Theorem 3.7 for compact space). Such $A^{*} \in \mathscr{K}_{o}(X)$ is also called as Fractal generated by the IF-IMS of multivalued intuitionistic fuzzy B-contractions (respectively multivalued intuitionistic fuzzy Edelstein contractions) and so called as Multivalued Intuitionistic Fuzzy Fractal on complete space (respectively compact space).

Corollary 3.9. [HB Theorem of IF-IMS in the Standard Intuitionistic Fuzzy Metric Spaces] Let $\left(X, M_{d}, N_{d}, *, \diamond\right)$ be a standard intuitionistic fuzzy metric space induced by the metric $d$, where $(X, d)$ is a complete metric space. Let $\left\{X ; f_{n}, n=1,2,3, \ldots, N_{o}\right.$; $\left.N_{o} \in \mathbb{N}\right\}$ be an IF-IMS of multivalued intuitionistic fuzzy B-contractions (respectively multivalued intuitionistic fuzzy Edelstein contractions). Then, there exists only one compact invariant set $A^{*} \in \mathscr{K}_{o}(X)$ of the IF-HB operator $\left(F^{*}\right)$ or, equivalently, $F^{*}$ has a unique fixed point namely $A^{*} \in \mathscr{K}_{o}(X)$.

Definition 3.10. The fixed point $A^{*} \in \mathscr{K}_{o}(X)$ of the IF-HB operator $F^{*}$ described in the Corollary 3.9 is called the Standard Multivalued Intuitionistic Fuzzy Fractal generated by the IF-IMS on the standard intuitionistic fuzzy metric space. 


\section{Remarks 3.11.}

1. If $B$ is a non-empty compact set in $\left(X, \tau_{d}\right)$, where $\tau_{d}$ is a topology induced by a metric $d$ on $X$, then $B$ is also a non-empty compact set in $\left(X, \tau_{\left(M_{d}, N_{d}\right)}\right)$, where $\tau_{\left(M_{d}, N_{d}\right)}$ is a topology induced by a standard intuitionistic fuzzy metric $\left(M_{d}, N_{d}\right)$ on $X$; and conversely.

2. Let $\left(X, M_{d}, N_{d}, *, \diamond\right)$ be a standard intuitionistic fuzzy metric space induced by the metric $d$ on $X$. Then, $A^{*}$ is a multivalued intuitionistic fuzzy attractor (multivalued intuitionistic fuzzy fractal) of the IF-IMS on $\left(X, M_{d}, N_{d}, *, \diamond\right)$ if and only if $A^{*}$ is a multivalued attractor (multivalued fractal) of the IFS on $(X, d)$.

\section{Collage Theorem for IF-IMS}

In Mathematics, the Collage theorem characterizes an iterated function system whose attractor is close, relative to the Hausdorff metric, to a given set. The IFS described is composed of contractions whose images, as a collage or union when mapping the given set, are arbitrarily close to the given set. It is typically used in fractal compression. In the literature, some important theorems related with metric fractals such as Collage Theorem is directly used in the distance concepts between the image pixels while processing the image. These fractal oriented concepts also are widely used in image processing, image denoising, image compression and etc. Mainly Hausdorff distance is applied in the image processing.

Many examples are constructed in fuzzy metrics and these concepts are employed for the fast detection of noisy pixels by taking advantage of the fuzzy metric properties [31]. Hence, we have the necessity to develop and prove the Collage Theorem for IF-IMS in the Standard Intuitionistic Fuzzy Metric Spaces in this section.

Theorem 4.1. [Collage Theorem for IF-IMS of Multivalued Intuitionistic Fuzzy B-Contractions] Let $\left(X, M_{d}, N_{d}, *, \diamond\right)$ be a standard intuitionistic fuzzy metric space induced by the metric $d$, where $(X, d)$ is a complete metric space. Let $\left(\mathscr{K}_{o}(X), H_{M}\right.$, $\left.H_{N}, *, \diamond\right)$ be the corresponding Hausdorff intuitionistic fuzzy metric space and $\left\{X ; f_{n}\right.$, $\left.n=1,2,3, \ldots, N_{o} ; N_{o} \in \mathbb{N}\right\}$ be an IF-IMS of multivalued intuitionistic fuzzy Bcontractions with the contractivity ratio $k$. Then for all $B \in \mathscr{K}_{o}(X)$ and $t>0$,

$$
\begin{gathered}
H_{M_{d}}\left(A^{*}, B, t\right) \geq H_{M_{d}}\left(B, F^{*}(B), t(1-k)\right) \\
\text { and } \\
H_{N_{d}}\left(A^{*}, B, t\right) \leq H_{N_{d}}\left(B, F^{*}(B), t(1-k)\right)
\end{gathered}
$$

where $F^{*}$ is the IF-HB operator and $A^{*}$ is the multivalued intuitionistic fuzzy attractor (multivalued intuitionistic fuzzy fractal) of the IF-IMS. 
Proof. Fix $t>0, k \in(0,1)$ and let $B \in \mathscr{K}_{o}(X)$. By the Theorem 2.8, we have

$$
\frac{t}{t+H_{d}\left(A^{*}, B\right)} \geq \frac{t(1-k)}{t(1-k)+H_{d}\left(B, F^{*}(B)\right)}
$$

and

$$
\frac{H_{d}\left(A^{*}, B\right)}{t+H_{d}\left(A^{*}, B\right)} \leq \frac{H_{d}\left(B, F^{*}(B)\right)}{t(1-k)+H_{d}\left(B, F^{*}(B)\right)} .
$$

Now by using the Proposition 2.16,

$$
\begin{aligned}
H_{M_{d}}\left(A^{*}, B, t\right) & =M_{H_{d}}\left(A^{*}, B, t\right) \\
& =\frac{t}{t+H_{d}\left(A^{*}, B\right)} \\
& \geq \frac{t(1-k)}{t(1-k)+H_{d}\left(B, F^{*}(B)\right)} \\
& =M_{H_{d}}\left(B, F^{*}(B), t(1-k)\right) \\
& =H_{M_{d}}\left(B, F^{*}(B), t(1-k)\right) .
\end{aligned}
$$

Similarly,

$$
\begin{aligned}
H_{N_{d}}\left(A^{*}, B, t\right) & =N_{H_{d}}\left(A^{*}, B, t\right) \\
& =\frac{H_{d}\left(A^{*}, B\right)}{t+H_{d}\left(A^{*}, B\right)} \\
& \leq \frac{H_{d}\left(B, F^{*}(B)\right)}{t(1-k)+H_{d}\left(B, F^{*}(B)\right)} \\
& =N_{H_{d}}\left(B, F^{*}(B), t(1-k)\right) \\
& =H_{N_{d}}\left(B, F^{*}(B), t(1-k)\right) .
\end{aligned}
$$

\section{Conclusion}

In this study, we defined the concept of multivalued fractals in intuitionistic fuzzy metric spaces via iterated multifunction system, which consists of finite number of intuitionistic fuzzy B-contractions and intuitionistic fuzzy Edelstein contractions. For that we proved the existence and uniqueness of multivalued fractals in a complete intuitionistic fuzzy 
metric space and a compact intuitionistic fuzzy metric space. Further, we discussed the Collage Theorem to analyze the multivalued intuitionistic fuzzy fractals in the standard intuitionistic fuzzy metric spaces. The multivalued intuitionistic fuzzy fractal theory will be useful in the area of image processing, pattern recognition, biomedical data analysis and so on.

\section{References}

[1] L.A. Zadeh, Fuzzy Sets, Information and Control 8 (1965) 338-353.

[2] K.T. Atanassov, Intuitionistic Fuzzy Sets, Fuzzy Sets and Systems 20 (1986) 87-96.

[3] I. Kramosil, J. Michalek, Fuzzy metrics and statistical metric Spaces, Kybernetika 11(5) (1975) 336-344.

[4] A. George, P. Veeramani, On some results in fuzzy metric spaces, Fuzzy Sets and Systems 64 (1994) 395-399.

[5] A. George, P. Veeramani, On some results of analysis for fuzzy metric spaces, Fuzzy Sets and Systems 90 (1997) 365-368.

[6] V. Gregori, A. Sapena, On fixed-point theorems in fuzzy metric spaces, Fuzzy Sets and Systems 125 (2002) 245-252.

[7] J.H. Park, Intuitionistic fuzzy metric spaces, Chaos, Solitons and Fractals 22 (2004) 1039-1046.

[8] C. Alaca, D. Turkoglu, C. Yildiz, Fixed points in intuitionistic fuzzy metric spaces, Chaos, Solitons and Fractals 29 (2006) 1073-1078.

[9] A. Mohamad, Fixed-point theorems in intuitionistic fuzzy metric spaces, Chaos, Solitons and Fractals 34 (2007) 1689-1695.

[10] B.B. Mandelbrot, The Fractal Geometry of Nature, W.H. Freeman and Company, New York, 1983.

[11] J.E. Hutchinson, Fractals and self similarity, Indiana University Mathematics Journal 30 (1981) 713-747.

[12] M. Barnsley, Fractals Everywhere, second ed., Academic Press, USA, 1993.

[13] M. Barnsley, Super Fractals, Cambridge University Press, New York, 2006.

[14] K. Falconer, Fractal Geometry: Mathematical Foundations and Applications, second ed., John Wiley \& Sons Ltd., England, 2003.

[15] G. Edgar, Measure, Topology, and Fractal Geometry, second ed., Springer, New York, 2008.

[16] J. Rodriguez-Lope, S. Romaguera, The Hausdorff fuzzy metric on compact sets, Fuzzy Sets and Systems 147 (2004) 273-283.

[17] J.Andres, J. Fiser, Metric and topological multivalued fractals, International Journal of Bifurcation and Chaos 14(4) (2004) 1277-1289. 
[18] J. Andres, J. Fiser, G. Gabor, K. Lesniak, Multivalued fractals, Chaos, Solitons and Fractals 24 (2005) 665-700.

[19] J. Andres, M. Rypka, Multivalued fractals and hyperfractals, International Journal of Bifurcation and Chaos 22(1) (2012) Article No.: 1250009.

[20] J. Fiser, Numerical aspects of multivalued fractals, Fixed Point Theory 5(2) (2004) 249-264.

[21] C. Chifu, A. Petrusel, Multivalued fractals and generalized multivalued contractions, Chaos, Solitons and Fractals 36 (2008) 203-210.

[22] S.L. Singh, Bhagwati Prasad, Ashish Kumar, Fractals via iterated functions and multifunctions, Chaos, Solitons and Fractals 39 (2009) 1224-1231.

[23] N.M.G. Al-Saidi, M. Rushdan Md. Said, A.M. Ahmed, IFS on the Multi-Fuzzy Fractal Space, International Journal of Computational and Mathematical Sciences 3(2) (2009) 82-86.

[24] N.M.G. Al-Saidi, M. Rushdan Md. Said, A.M. Ahmed, About Fuzzy Fixed Point Theorem in the Generalized Fuzzy Fractal Space, Far East Journal of Mathematical Sciences 53(1) (2011) 53-64.

[25] D. Easwaramoorthy, R. Uthayakumar, Analysis on Fractals in Fuzzy Metric Spaces, Fractals 19(3) (2011) 379-386.

[26] R. Uthayakumar, D. Easwaramoorthy, Hutchinson-Barnsley Operator in Fuzzy Metric Spaces, World Academy of Science, Engineering and Technology 56 (2011) $1372-1376$.

[27] D. Easwaramoorthy, R. Uthayakumar, Intuitionistic Fuzzy Fractals on Complete and Compact Spaces, in: P. Balasubramaniam (Ed.), Control, Computation and Information Systems, Communications in Computer and Information Science, Springer-Verlag Berlin Heidelberg, 140, 2011, pp. 89-96.

[28] R. Uthayakumar, D. Easwaramoorthy, Analysis on Fractals in Intuitionistic Fuzzy Metric Spaces, World Academy of Science, Engineering and Technology 68 (2012) 630-636.

[29] R. Uthayakumar, D. Easwaramoorthy, Hutchinson-Barnsley Operator in Intuitionistic Fuzzy Metric Spaces, World Academy of Science, Engineering and Technology 68 (2012) 676-683.

[30] V. Gregori, S. Romaguera, P. Veeramani, A note on intuitionistic fuzzy metric spaces, Chaos, Solitons and Fractals 28 (2006) 902-905.

[31] V. Gregori, S. Morillas, A. Sapena, Examples of fuzzy metrics and applications, Fuzzy Sets and Systems 170 (2011) 95-111.

[32] B. Schweizer, S. Sklar, Statistical metric spaces, Pacific Journal of Mathematics 10 (1960) 314-334. 


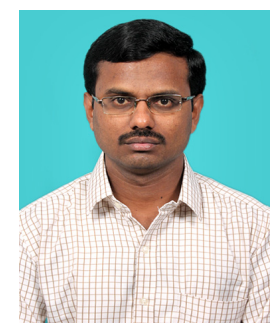

D. Easwaramoorthy was born in Rasipuram, Namakkal, Tamil Nadu, India; in 1986. He received the M.Sc. Degree in Mathematics from the Bharathidasan University, Tiruchirappalli, India in 2008 and the Ph.D. Degree in Mathematics from The Gandhigram Rural Institute - Deemed University, Gandhigram, India in 2013. Currently, he is an Assistant Professor in Mathematics, School of Advanced Sciences, VIT University, Vellore, India. His research interests include Fuzzy Fractal Analysis and Signal \& Image Analysis.

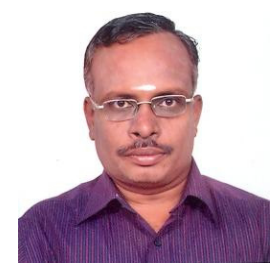

R. Uthayakumar was born in Dindigul, India; in 1967. He received the Ph.D. Degree in Mathematics from The Gandhigram Rural Institute - Deemed University, Gandhigram, India; in 2000. He is currently a Professor in Mathematics in The Gandhigram Rural Institute - Deemed University, Gandhigram, India. His major research interests include Mathematical Modeling, Fractal Analysis, Inventory Management \& Control, Supply Chain Systems and Biomedical Applications. 
